CERN-PPE/94-196

30 November $\overline{994}$

Sue 9450

\title{
APPLICATIONS OF GASEOUS PARTICLE DETECTORS IN PHYSICS AND MEDICINE
}

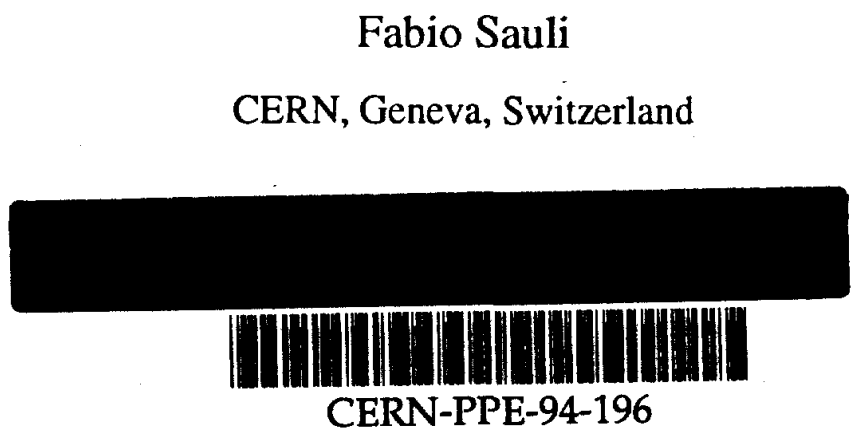

\section{ABSTRACT}

The multi-wire proportional chamber, introduced in 1967 by Georges Charpak (recipient of the 1992 Nobel prize for physics) allows to achieve high-rate, fully electronics detection and localization of ionizing radiation. The myriad of devices inspired by this initial work generated a revolution in the conception of detectors for elementary particle physics experiments; examples are the time projection chamber, the drift chamber, the micro-strip gas chamber. After a brief introduction on the basic operating principles of the device, I will describe several examples of application of advanced gas detectors in medicine and biology and analyze the operating characteristics that make the new devices attractive when confronted with classic detectors. 


\title{
Applications of Gaseous Particle Detectors in Physics and Medicine
}

\author{
Fabio Sauli \\ CERN, CH-1211 Geneva, Switzerland
}

\begin{abstract}
The multi-wire proportional chamber, introduced in 1967 by Georges Charpak (recipient of the 1992 Nobel prize for physics) allows to achieve high-rate, fully electronics detection and localization of ionizing radiation. The myriad of devices inspired by this initial work generated a revolution in the conception of detectors for elementary particle physics experiments; examples are the time projection chamber, the drift chamber, the micro-strip gas chamber. After a brief introduction on the basic operating principles of the device, I will describe several examples of application of advanced gas detectors in medicine and biology and analyze the operating characteristics that make the new devices attractive when confronted with classic detectors.
\end{abstract}

\section{INTRODUCTION}

Ionization, proportional and Geiger counters have been used for decades for the detection of radiation. Large area, fast position-sensitive gaseous detectors based on the multiwire proportional chamber (MWPC) have been developed, primarily to satisfy the stringent demands of high energy physics. MWPCs and their offspring represent in many cases unique tools for detection and localization of radiation; their diffusion however in applied fields has been somewhat hindered by the fact that they are complex and often fragile instruments requiring dedicated operators. The continuing efforts to increase the ruggedness and reliability of the devices, and the development of performing wireless detectors (parallel plate and micro-strip gas chambers) permit to foresee an increasing use of gaseous detectors in applied research. This paper describes some examples of use of gaseous devices in medicine and biology.

\section{THE MULTIWIRE PROPORTIONAL CHAMBER}

The multi-wire proportional chamber, developed in the late sixties by Georges Charpak (1), has thereafter been refined and diversified in a large variety of devices exploiting detailed gas properties and improving on performances. A comprehensive description of these detectors can be found in dedicated review articles and books (2-5). In recognition of the large impact on science of the new technology, Charpak was awarded the 1992 Nobel prize for physics.

In its basic configuration, the MWPC consists of a set of thin, parallel anode wires stretched between two cathode planes (Fig. 1). Application of a symmetric difference of potential between anodes and cathodes creates the field shown in the figure. Electrons released by ionization in the gas drift towards the anodes and experience ionizing collisions in the high electric field approaching the thin wires; the ensuing avalanche multiplication imparts a large gain to the charge signal, by a factor as large as $10^{6}$ or more. 


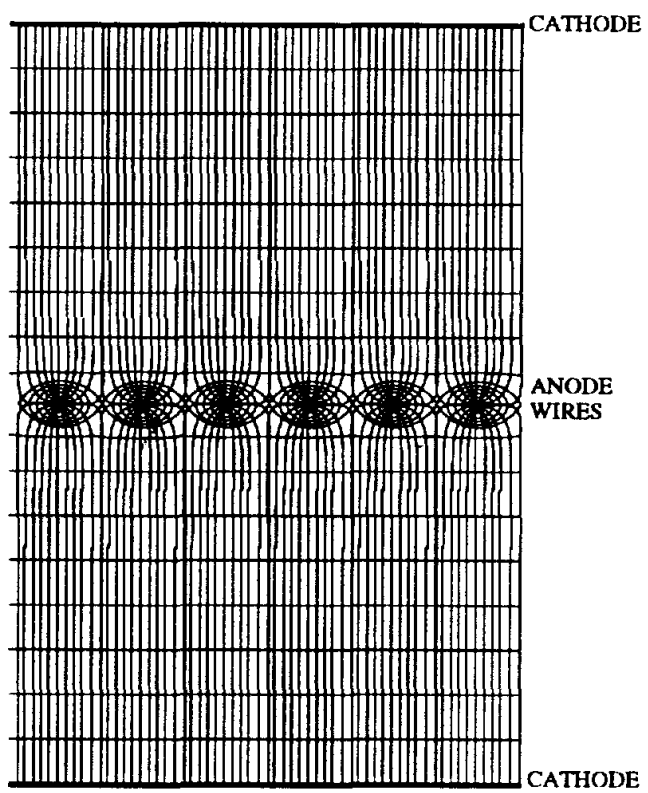

FIGURE 1: Schematics of the multiwire proportional chamber and of the applied field.

Various methods can be used to achieve localization of the ionization: detection and counting of pulses over threshold on each anode wire; measurement of the centre of gravity of signals induced by the avalanches on cathode strips; measurement of the collection or drift time of charges. With anode and cathode wire planes segmented and equipped with recording electronics, MWPCs allow to determine with sub-millimetre accuracy the co-ordinates of an avalanche generated by the multiplication processes in the gas; as shown in Fig. 2, the induced signals on cathodes, as well as the signals on anodes, can be detected and used to obtain a two-dimensional localisation of the original ionization.

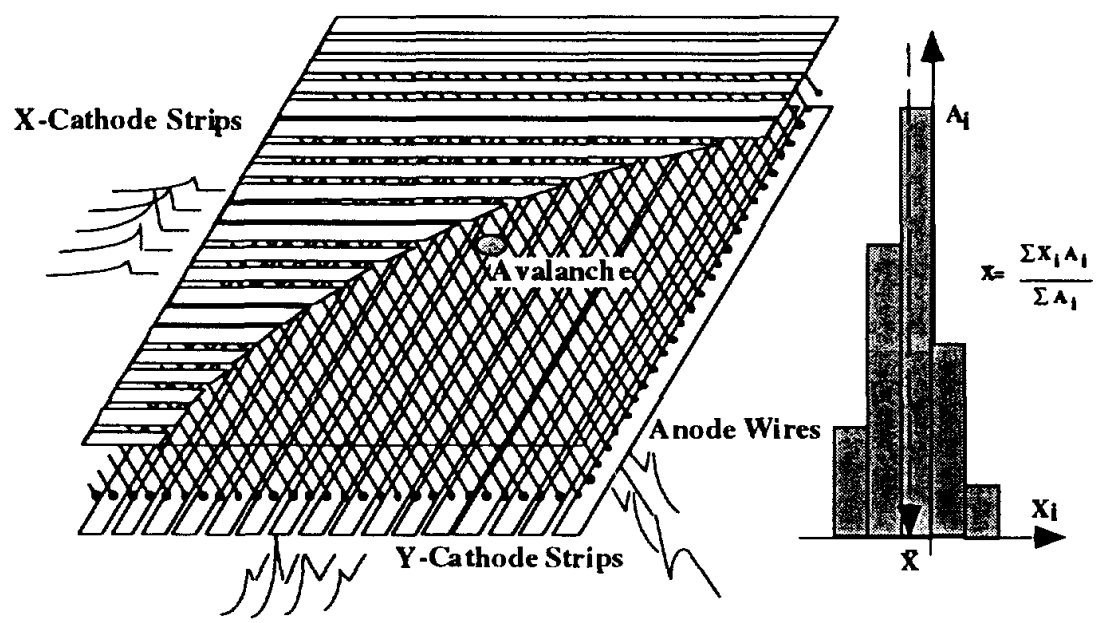

FIGURE 2: Centre-of gravity method for co-ordinate measurement in MWPC. 
In medicine and biology, a widely used readout method makes use of delay lines, transforming the space co-ordinate of the induced signals on cathodes into time delays, the pulse detected on the anode wire plane being used to provide the reference time (6). The rate limitations and single hit capability of such readout method are compensated by the great simplicity and low cost. For recent reviews of applications of MWPCs see for example (7-9).

\section{MEDICAL DIAGNOSTICS: DIGITAL RADIOGRAPHY}

Substantial improvements over standard diagnostics methods can be obtained with gaseous detectors used as X-ray imagers; in many cases, comparable contrast levels can obtained at a much reduced dose, with obvious advantage for the patients. To achieve good detection efficiency for hard X-rays, one has to use rather thick conversion layers and possibly high pressures: xenon mixtures are preferred to reduce the physical thickness of the detector: Various methods have been devised to eliminate the dispersive effects of thick conversion layer for non-parallel $X$-ray beams (the parallax error). In the digital radiography scanner (10) shown schematically in Fig. 3, a thin collimated X-ray beam is sent through the body and the transmitted radiation is detected in a specially designed MWPC. Generator and detector move together vertically to realize a body scan; in order to allow fast imaging, the simplest method of electronics detection (counting on fast scalers) is used to permit high acquisition rates.

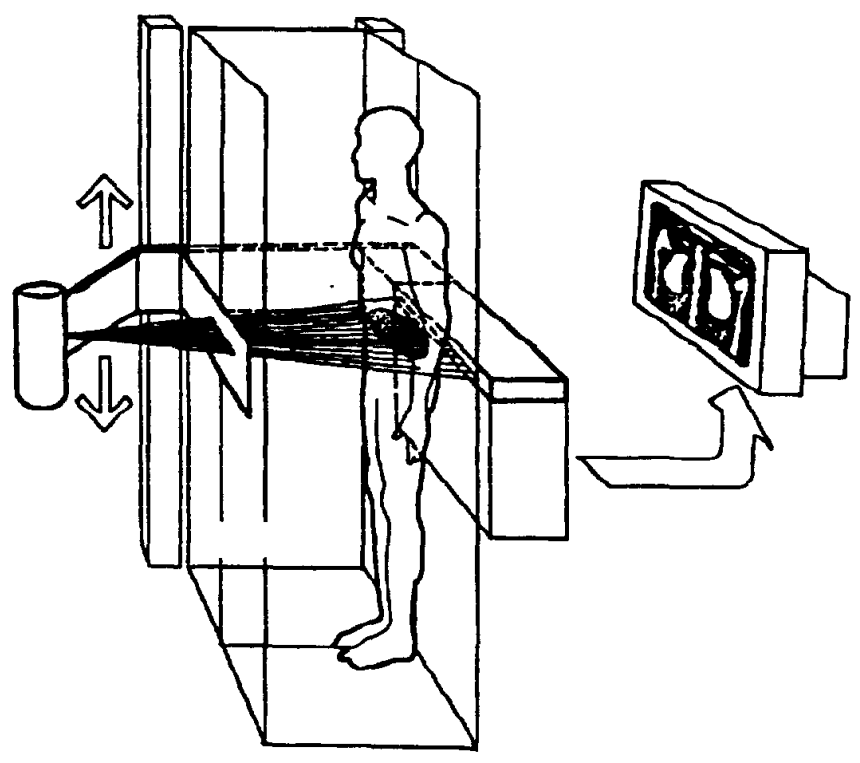

FIGURE 3: Digital radiography scanner with a MWPC detector.

The particular conception of the MWPC detector (Fig. 4) with non-parallel anode wires aiming at the focal point of the generator allows to use a thick absorption volume without a parallax error; the uniformity of gain along the anodes is guaranteed by an increasing gap between anode wires and cathode planes. 


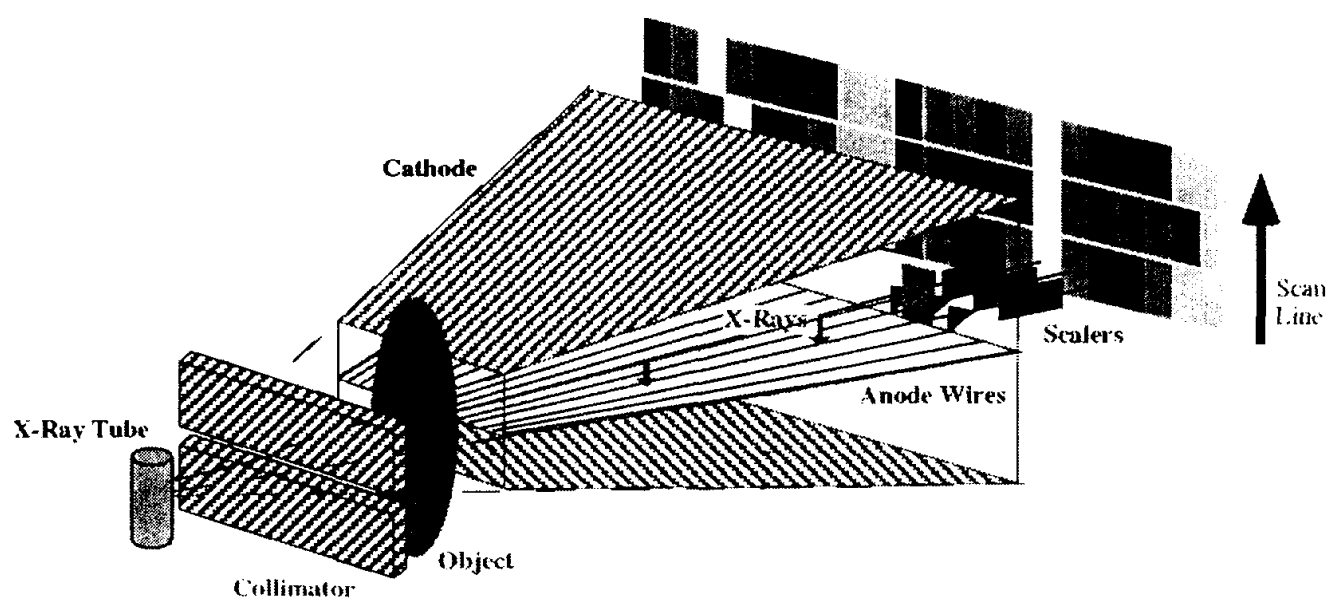

FIGURE 4: Schematics of the MWPC detector for the digital radiography.

Digital radiography systems as described above are presently evaluated in hospital environment, in Russia and in the West; a detailed comparison of performances as a function of dose to the patient clearly demonstrates the advantages of the electronics system over classic photographic plates, particularly for low-dose exposures (11). Fig. 5 shows an example of digital radiography of a woman's pelvic region (hysterosalpingography), obtained at an entrance skin dose of $0.030 \mathrm{mGy}$; a contrast medium was injected to enhance the features under study (a partially obstructed fallopian tube). Even at low doses, the counting rate capability of the device has to be large in order to decrease the exposure time and to reduce artefacts due to the movements of the body or of internal organs during the scan; using fast scalers, the counting rate in the present design is limited to $600 \mathrm{kHz}$ per wire.

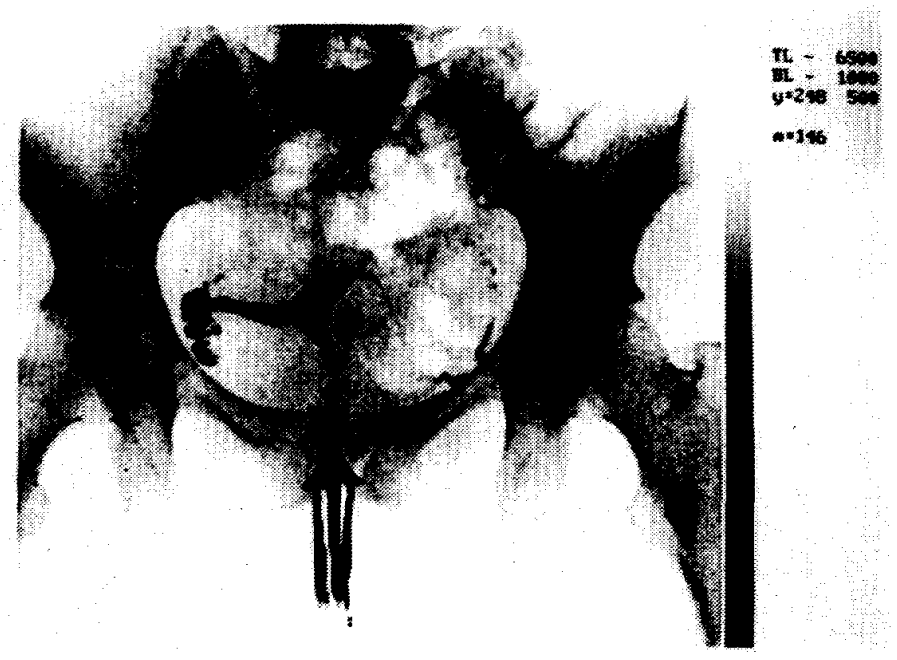

FIGURE 5: Digital radiography of the pelvis. 


\section{ANGIOGAPHY WITH A GASEOUS DETECTOR}

In conventional coronary angiography, a contrast medium (iodine) is injected in coronary arteries and a sequence of X-ray absorption images is taken at very high speed. The method provides very good quality images, but involves a serious risk for the patient because, to avoid dilution, the iodine is injected as close as possible to the coronary with a catheter introduced from the femoral artery. A less invasive method, with the contrast medium injected in a peripheral vessel would be a progress, but the resulting dilution results in a substantial reduction of contrast. One way to improve the contrast is to realize the absorption radiography at two $\mathrm{X}$-ray energies, one just below and the other above the iodine $\mathrm{K}$-shell absorption edge (33.17 keV), and logarithmically subtract the images to eliminate the contributions of tissues surrounding the vessels. Exposures have to be simultaneous and very fast (typically $1 \mathrm{~ms}$ per line, or less than half a second per frame) and images should be available in digital form for the subtraction. The requirements can be met by the use of a synchrotron radiation source and of a gaseous detector with fast digital readout $(12,13)$.

Fig. 6 shows the set-up used. The wide-band X-ray beam produced by the synchrotron radiation source at DESY is filtered by a pair of monochromators and split in two beams of energies $300 \mathrm{eV}$ apart, below and above $33.17 \mathrm{keV}$; the narrow beams overlap in the region under examination. Two detectors measure the transmitted intensity on a line, and to realize a two-dimensional scan the patient is translated along the vertical axis.

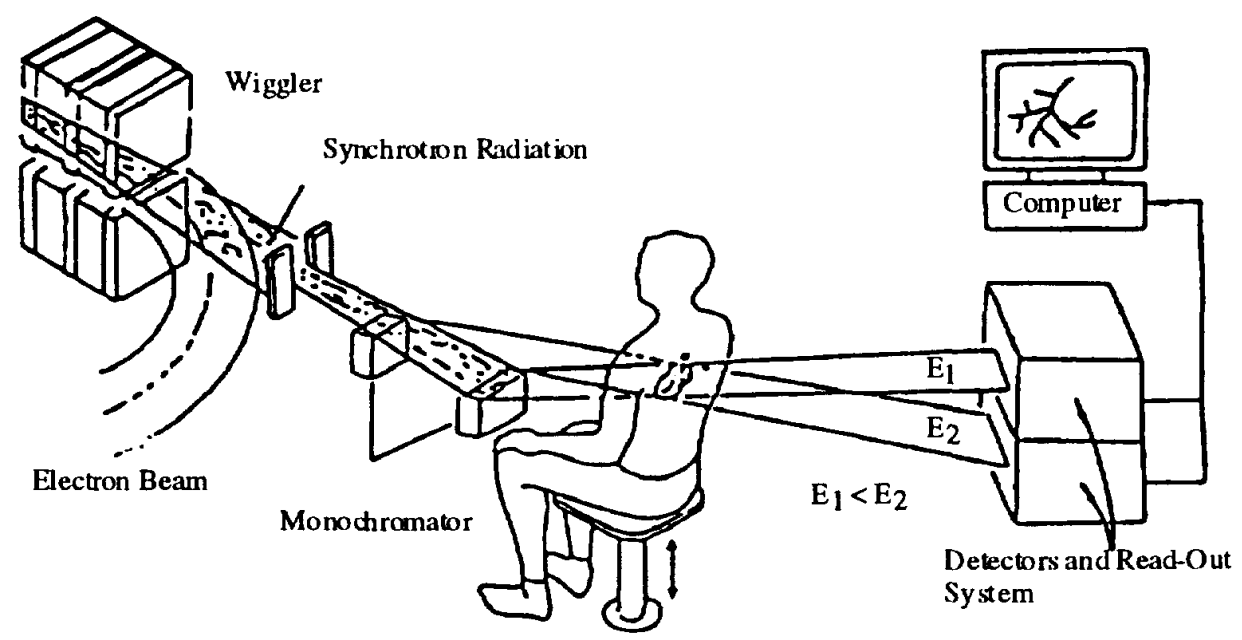

FIGURE 6: Dual beam scanning apparatus used for coronary subtraction angiography.

Because of the stringent speed requirements, the data acquisition has to be very fast and this implies the use of high intensity beams, typically $10^{11}$ photons $\mathrm{mm}^{-2} \mathrm{~s}^{-1}$. As this exceeds by orders of magnitude the rate capability of gaseous detectors with individual counting, the authors have used a device operated in the ionization mode. The detector is shown schematically in Fig. 7, and consists in two independent multiwire ionization chambers mounted back to back. 


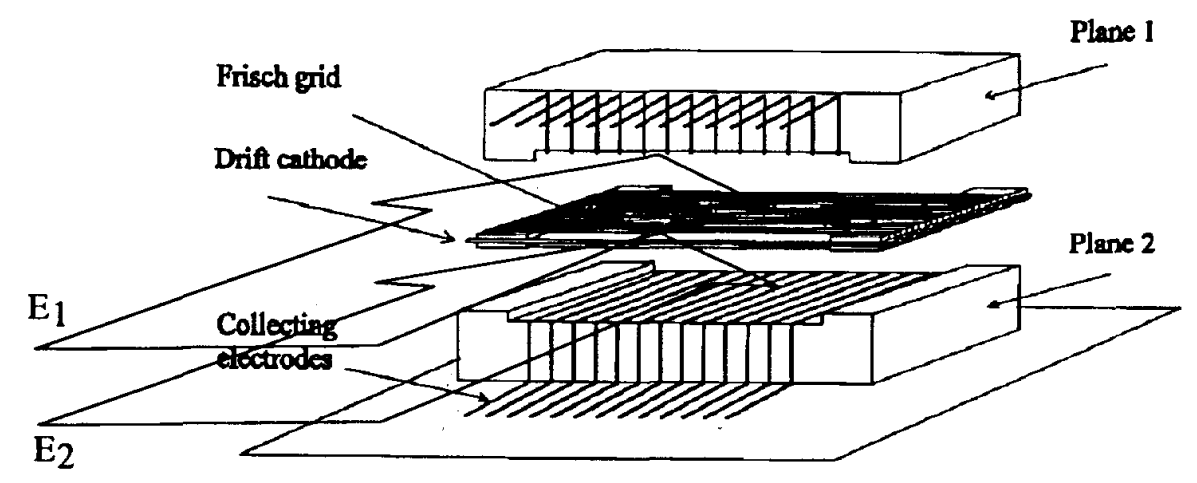

FIGURE 7: Dual-beam multiwire ionization detector.

With a pixel size of 0.5 by $0.5 \mathrm{~mm}^{2}$ and a field of about 12 by $12 \mathrm{~cm}^{2}$, the system scans a full image in about $250 \mathrm{~ms}$ ( $1 \mathrm{~ms}$ per line); an example of raw and subtracted coronary angiography realized with the set-up is shown in Fig. 8 (14):

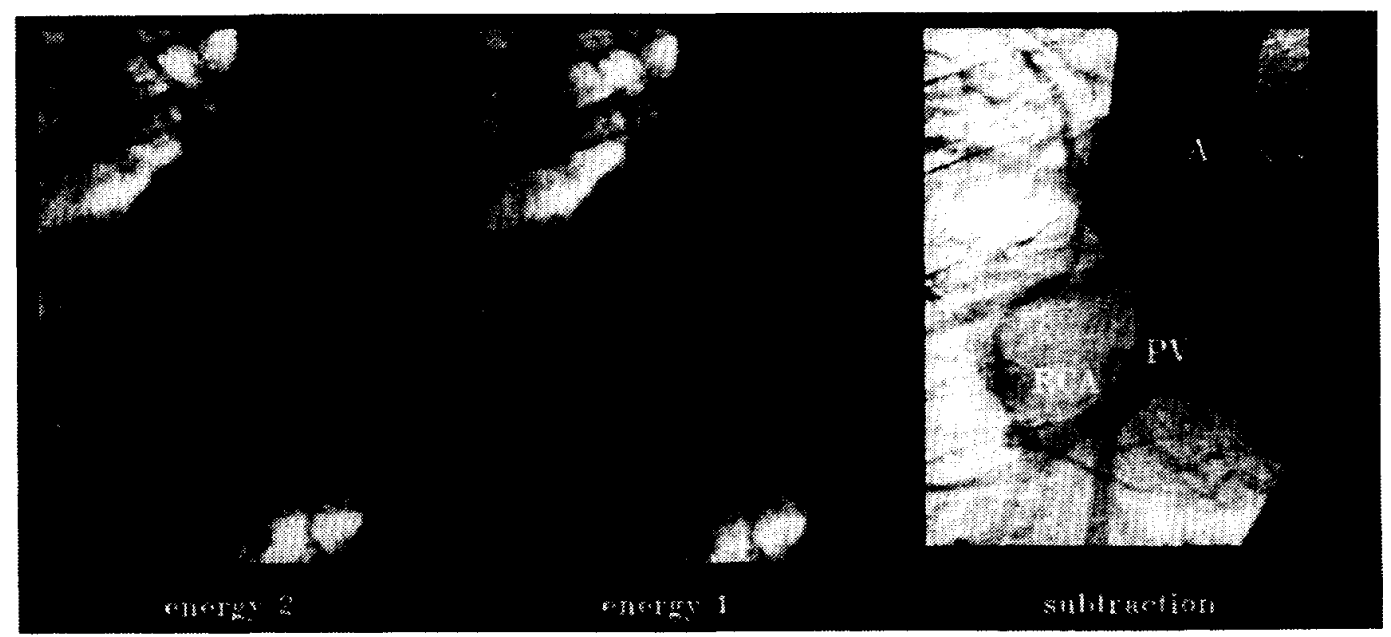

FIGURE 8: Single-energy (left) and subtracted (right) coronary.

\section{APPLICATIONS IN BIOLOGY: RADIO-CHROMATOGRAPHY}

Charge multiplication is exploited in gaseous counters for amplification of the ionization signal; photons are also however copiously emitted in the avalanche as a result of molecular excitation and recombination processes. Addition of low ionization threshold vapours, together with the use of a parallel plate geometry (as against a MWPC structure), largely enhance the emission in a spectral region convenient for optical detection. The structure of an optical imaging chamber (15) is shown schematically in Fig. 9; electrons released in a gas by ionizing radiation drift under the influence of an electric field towards a region of very high field between two semi-transparent grids. During the avalanche development, photons are emitted and transmitted through a window; an image intensifier is used to 
amplify the signal and bring it up to a level compatible with the sensitivity of a solid state camera. The image can be recorded directly or after digitization. The simplicity and the high granularity of the device has suggested the application in fields where these performances largely compensate for the modest data acquisition rate obtainable, for example to map the two-dimensional activity distribution in anatomical samples marked with radioactive labellers (16). Fig. 10 shows examples of activity distributions in anatomical samples (brain slices of male and female rats labelled with tritiated vasopressin recorded with the imaging chamber (17); the study aims at establishing the effects of hormones release at the early stages of development on the sex of mature animals.
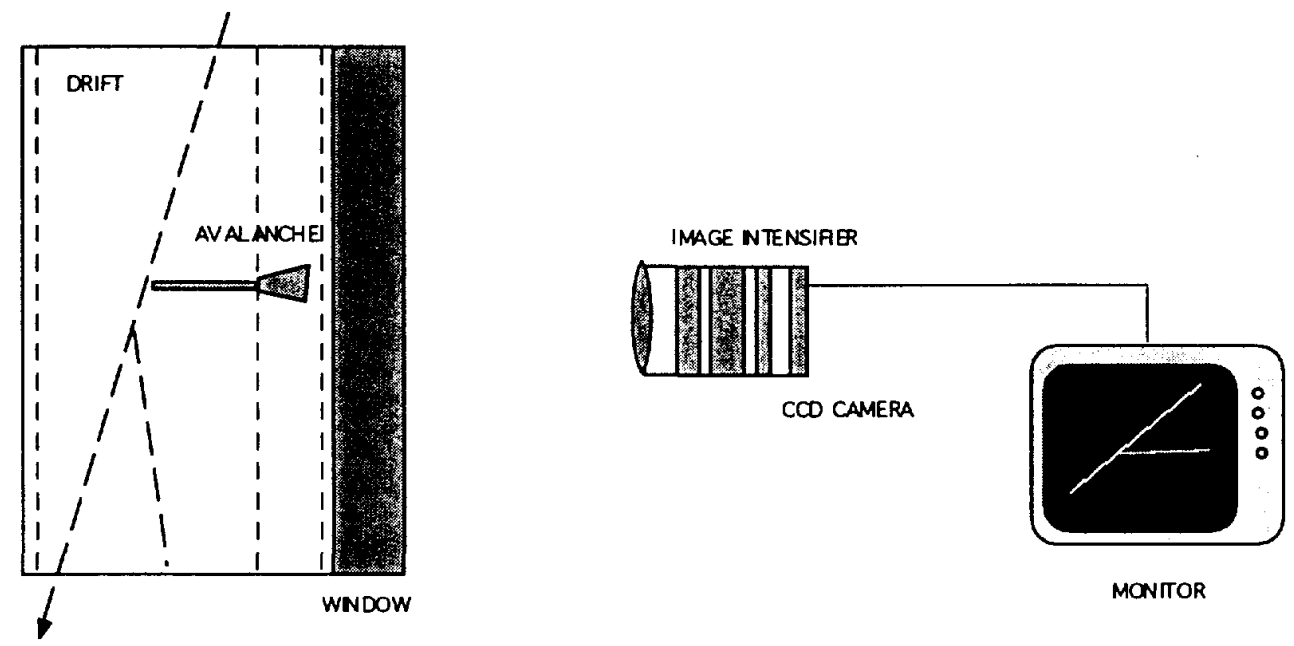

FIGURE 9: The imaging chamber.
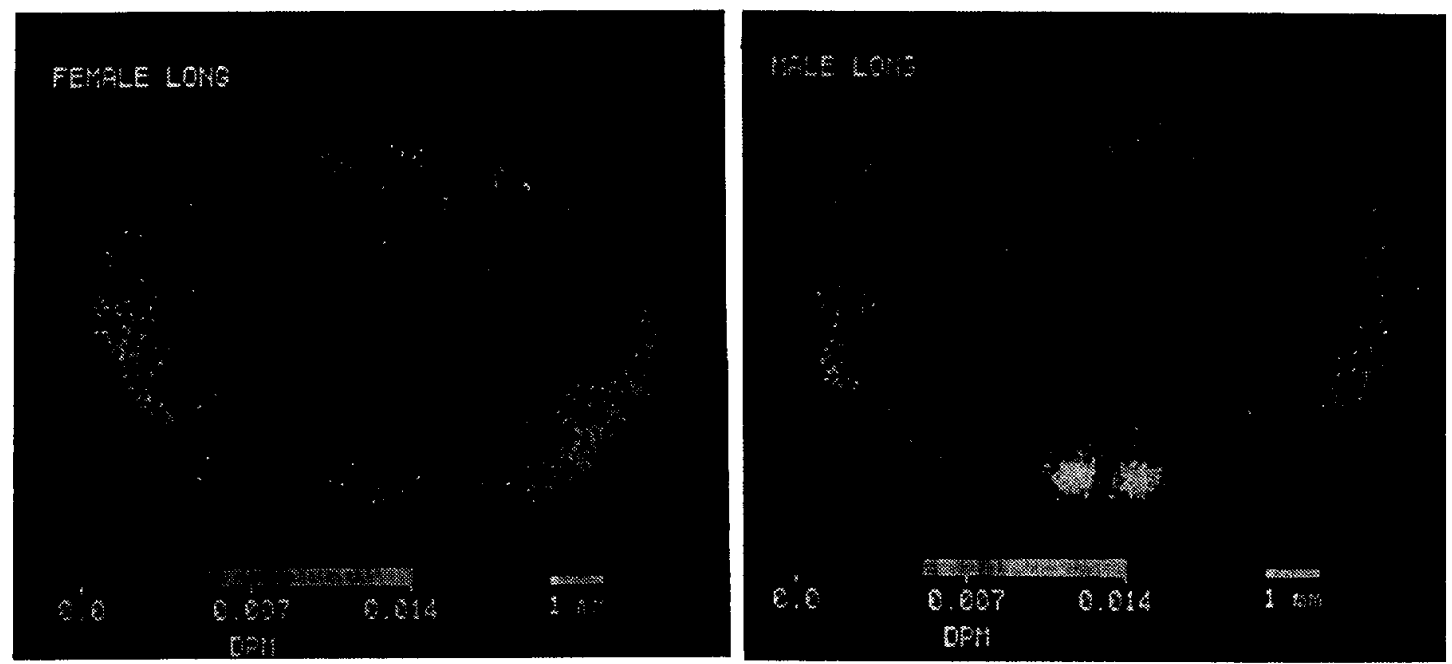

FIGURE 10: Examples of radio-chromatography of tritiated slices of anatomical samples (brain of female and male rats). 
Due to the sensitivity to individual radioactive decays, comparable contrasts are obtained with exposure times one or two order of magnitude shorter with the gaseous device as compared to film. This obviously allows the realization of exposure-intensive studies. Another advantage of the gas detector over conventional contact auto-radiography with is the linearity of the response over an extended range of activity, allowing quantitative analysis in a single exposure.

\section{NEW DETECTORS : THE MICRO-STRIP CHAMBER}

Multiwire proportional chambers, although very powerful, suffer from a serious intrinsic limitation: at high radiation rates, the production of positive ions by the avalanches, coupled with their low mobility, results in the build-up of a space charge that interferes with the counting action. In standard operating conditions, a MWPC begins to be inefficient at rates above $10^{4} \mathrm{~mm}^{-2} \mathrm{~s}^{-1}$. As seen above, this can be a serious limitation for medical diagnostics requiring individual detection of events. A novel device, the micro-strip gas chamber, MSGC (16) holds very promising characteristics in terms of improved resolution and rate capability. The MSGC has received a lot of interest in particle physics in view of applications at high luminosity accelerators .

The basic structure of a MSGC is shown in Fig. 11. Thin, metallic parallel strips are laid on an insulating support using high precision photo-lithography; connected alternatively to positive and negative potentials, the strips act as a multi-anode proportional counter. Typical strip width is $10 \mu \mathrm{m}$ and $100 \mu \mathrm{m}$ for anodes and cathodes, respectively, at a distance centre to centre of $100 \mu \mathrm{m}$.

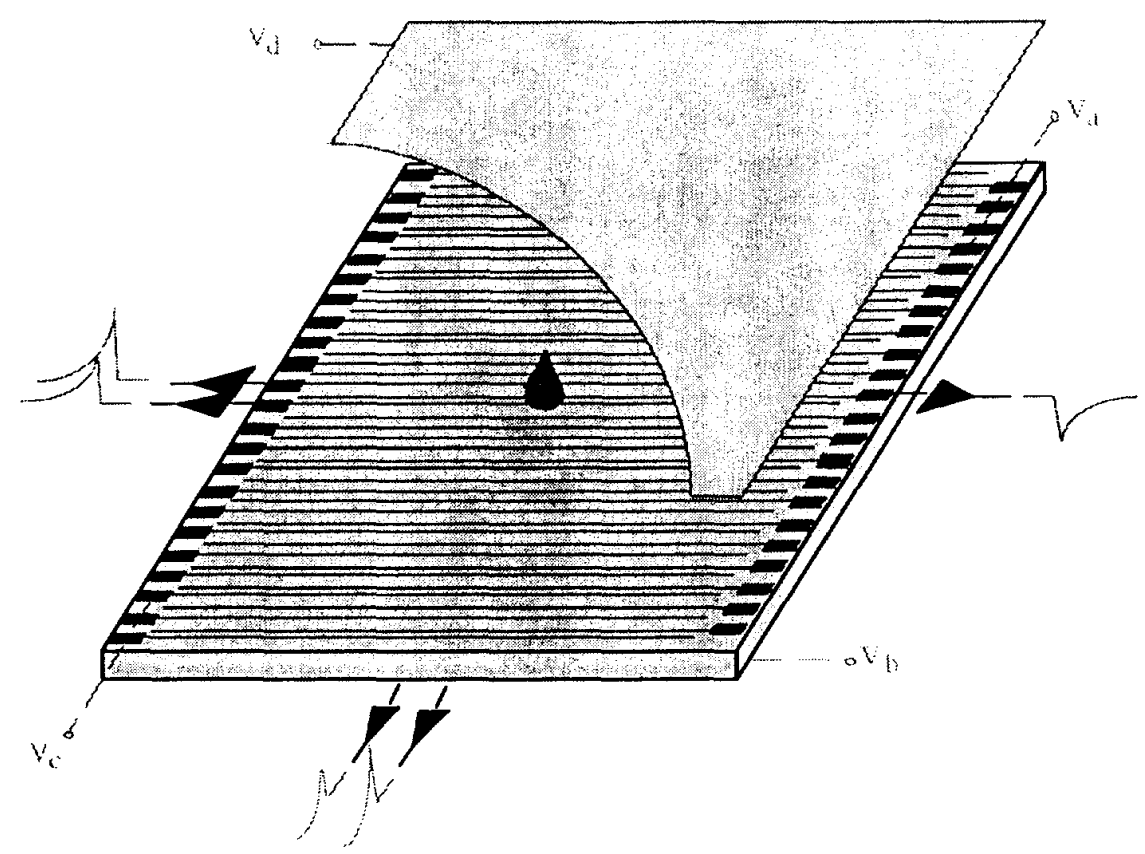

FIGURE 11: Schematics of the micro-strip gas chamber. 
As shown in the figure, the development of an avalanche generates a negative signal on the anode strip and positive induced signals on the neighbouring cathodes; using a thin foil as support, one can also detect induced pulses on the back plane thus permitting to realize a projective two-dimensional readout much as in the MWPC but at a scale an order of magnitude smaller. Several insulating materials have been used as substrate for manufacturing MSGCs, such as glass, plastic, silicon, sapphire. For operation at high rates, and in order to avoid the accumulation of ions in the interstice between strips, slightly conducting supports have to be used, with surface resistivity around $10^{15} \mathrm{ohm} / \mathrm{square}$; Fig. 12 provides a summary of rate capability measurement (gain as a function of flux) for several substrates, regular boro-silicate glass and special semi-conducting glass (19). For comparison, the rate capability of a standard MWPC is also shown; all measurements are referred to the same avalanche size $\left(3 \times 10^{5}\right.$ electrons $)$.

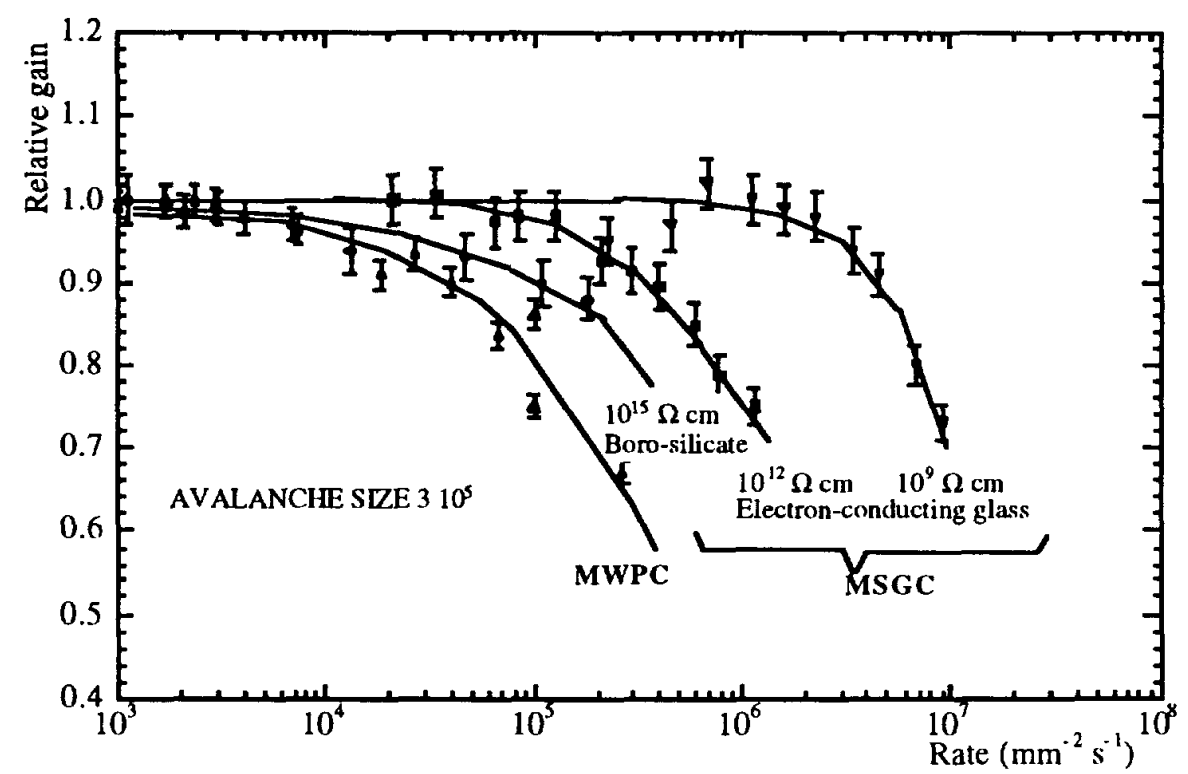

FIGURE 12: Rate capability of the MWPC and of MSGCs manufactured on substrates with several values of bulk or surface resistivity.

The intrinsic position accuracy of MSGC, measured for soft X-rays or for charged particles perpendicular to the plates, is of around $30 \mu \mathrm{m} \mathrm{rms,} \mathrm{obtained}$ recording the charge on cathode strips and computing its centre of gravity. This makes it a very attractive choice for applications where high rate and good space resolutions are required; this is the case for example for the digital radiography system described in a previous section. An instrument based on the MSGC as detector is presently under development (20). 


\section{REFERENCES}

1. Charpak, G., Bouclier, R., Bressani, T., Favier, J., and Zupancic, C., Nucl. Instrum. Methods 62 (1968) 235.

2. Charpak, G., Ann. Rev. Nucl. Sci. 20 (1970) 195.

3. Sauli, F., Principles of operation of Multiwire proportional and drift chambers, CERN 77-09 (1977).

4. Charpak, G.. and Sauli, F., Ann. Rev. Nucl. Sci. 34 (1984) 285.

5. Blum,W., and Rolandi, G., Particle detection with drift chambers (SpringerVerlag 1993).

6. Grove, R., Lee, K., Perez-Mendez,V., and Sperinde, J., Nucl. Instrum.

Methods 89 (1970) 257.

7. Sauli, F., Nucl. Instr. Methods A323 (1992) 1.

8. Bateman, J.E., Nucl. Sci. Appl. 3 (1988) 59.

9. Ott, R.J., Eur. J. Nucl. Medicine 20 (1993) 348.

10. Babichev, A.E., Baru, S.E., Gusev, V.V., A.G. Khabakhpasev, G.M.

Kolachev, G.A. Savinov, L.I. Shekhtman, Sidorov, V.A., and Volobiev, A.I., Nucl. Instr. Methods A310 (1991) 449.

11. Martínez-Dávalos, A., Speller, R.D., Horrocks, J.A., Miller, D.J., Baru, S.E., Khabakhpasev, A.G., Ponomarev, O.A., and Shekhtman, L.I., Phys. Med. Biol. 38 (1993) 1419.

12. Besch, H.J., Bode, E.J., Menk, R.H., Schenk, H.W., Tafelmeier, U., Walenta, A.H., and Xu, H.Z., Nucl. Instrum. Methods A310 (1991) 446.

13. Besch, H.J., Dix, W.R., Großmann, U., Heuer, J., Langer, R., Lohmann, M., Menk, R.H., Schenk, H.W., Tafelmeier, U., Wagener, M., Walenta, A.H., and Xu, H.C., Phys. Medica 9 (1993) 171.

14. Dix, W.R., Gaeff, W., Illing, G., Menk, R.H., Reime, B., Schildwächter, L., Tafelmeier, U., Besch, H.J., Lohmann, M., Walenta, A.H., Hamm, C.W., Meinertz, T., Rust, C., and Kupper, W., DESY SR 94-01 (1994).

15. Charpak, G., Fabre, J.P., Sauli, F., Suzuki, M., and Dominik, W., Nucl. Instrum. Methods A258 (1987) 177.

16. Dominik,W., Zaganidis, N., Astier, P., Charpak, G., Santiard, J.C., Sauli, F. Tribollet, E., Geissbühler, A., and Townsend, D., Nucl. Instr. Methods A278 (1989) 779.

17. Tribollet, E., Dreifuss, J.J., Charpak, G., Dominik, W., and Zaganidis, N., Proc. Natl. Acad. Sci. USA 88 (1991) 1466.

18. Oed,A., Nucl. Instrum. Methods A263 (1988) 351.

19. Bohm, J., Bouclier, R., Capeáns, M., Garabatos, Ch., Manzin, G., Million, G., Sauli, F., Temmel, T., and Shekhtman, L.I., CERN-PPE/94-115 (1994). Subm. Nucl. Instrum. Methods.

20. Minakov, G.D., Pestov, Yu.N., Prokopenko, V., and Shekhtman, L.I., Nucl. Instrum. Methods A326 (1993) 566. 